\title{
Transposition
}

Musique et Sciences Sociales

$1 \mid 2011$

Polyphonie et société

\section{Pour une musicologie de l'analyse plurielle.}

Entretien avec Jean Michel Bardez

Towards a musicology of multiple analyses

Julien Ségol

\section{(2) OpenEdition}

Journals

Édition électronique

URL : http://journals.openedition.org/transposition/314

DOI : 10.4000/transposition.314

ISSN : 2110-6134

Éditeur

CRAL - Centre de recherche sur les arts et le langage

\section{Référence électronique}

Julien Ségol, « Pour une musicologie de l'analyse plurielle. », Transposition [En ligne], 1 | 2011, mis en

ligne le 01 février 2011, consulté le 01 mai 2019. URL : http://journals.openedition.org/

transposition/314; DOI : 10.4000/transposition.314

Ce document a été généré automatiquement le 1 mai 2019.

\section{(c) (†) (2)}

La revue Transposition est mise à disposition selon les termes de la Licence Creative Commons Attribution - Partage dans les Mêmes Conditions 4.0 International. 


\title{
Pour une musicologie de l'analyse plurielle.
}

\author{
Entretien avec Jean Michel Bardez \\ Towards a musicology of multiple analyses
}

Julien Ségol

\section{Biographie}

1 Titulaire de prix du CNSMDP (classes de F. Lengelé, G. Hugon, B. de Crépy, R. Boutry, C. Ballif, O. Messiaen, M. Philippot) ainsi que d'un Doctorat en littérature comparée (Université de Paris X), Jean Michel Bardez est président de la Société Française d'Analyse Musicale (depuis 1992) et enseigne l'écriture-analyse-composition au Conservatoire $\mathrm{H}$. Berlioz (Paris) où il est également Conseiller aux Etudes. Son parcours et sa formation pluridisciplinaires font de lui une figure atypique de la vie musicale/musicologique française : explorant les voies de la création artistique, de la composition (pièces pour orchestre, ensembles, Voix, orgue etc ...) à l'improvisation - piano, orgue -, ou au dessin, à la peinture, il mène aussi une importante activité de recherche (contribution à la réalisation d'une vingtaine de congrès et colloques) d'édition (trois livres sur le XVIII siècle, une cinquantaine d'ouvrages pédagogiques, de multiples articles et préfaces, une Collection commune à six éditeurs, plusieurs collections de livres et de partitions en collaboration, éditeur de l'oeuvre du compositeur N. Obouhow aux Ed. H. Lemoine...). Membre du comité éditorial de la revue Analyse Musicale, puis, de la revue Musurgia et de la revue Internet Musimediane, il est également impliqué aux éditions Delatour, dans le développement d'une collection à vocation interdisciplinaire (arts visuels et sonores) : Transversales.

\section{De l'héritage des pairs : rénover la discipline}

JULIEN SÉgoL - L'année 2008 a été l'occasion d'événements significatifs pour la musicologie en France, avec la tenue de deux colloques majeurs : le premier revenait sur la 
grande entreprise dirigée par J.-J. Nattiez, L'Encyclopédie pour le XXIe siècle, pour proposer un «bilan critique" », cependant que l'on dressait en Sorbonne un état des lieux des «ancrages théoriques et du rayonnement international » de la sociologie de la musique en France $^{2}$. II semble qu'en termes de bilan un premier constat s'impose: la prise de conscience patente de la nécessité de décloisonner un champ disciplinaire encore trop peu affranchi de l'héritage du XIXe siècle. Vous-même, président de la Société Française d'Analyse Musicale, en appelez à la pratique d'une « analyse plurielle ». Est-ce là un constat unanime?

Jean Michel Bardez - Dans un livre consacré aux relations entre philosophes, encyclopédistes, théoriciens et musiciens au XVIII ${ }^{\mathrm{e}}$ siècle $(1980)^{3}$, j'avais suggéré un plan d'analyse plurielle qui supposait une série ouverte de «balayages » successifs appliqués à une «œuvre» (acoustique, formel, timbrique, rythmique, historique, sémiotique, sociologique, esthétique etc.) dont chacun reprendrait les "résultats » précédents en les intégrant et densifiant peu à peu la représentation de la musique ainsi considérée. C'est dire à quel point j'étais disposé à suivre très attentivement la séance inaugurale que nous avions été plusieurs à préparer avec le plus grand intérêt, lors du premier Congrès Européen d'Analyse Musicale, à Colmar (1989). La conscience de la possibilité d'une multiplicité des " accès » interprétatifs s'était en effet manifestée tout particulièrement à travers neuf propositions d'analyses de La terrasse des audiences $d u$ clair de lune, de Debussy. Cette aventure originale, qui avait marqué les esprits d'entrée de jeu, avait été présentée comme un jalon pour une méthodologie comparée, que Jean Molino avait qualifiée de «méta-analyse, conduisant à une connaissance plus fine de l'objet musical étudié » (Analyse Musicale $\mathrm{N}^{\circ} 16$ ). À une telle expérience avait succédé, lors du second Congrès Européen de Trento, une session comportant plusieurs propositions d'analyse de diverses exécutions chantées d'une « même » cadenza d'un air tiré de Lucia di Lammermoor de Donizetti. À l'ouverture du troisième Congrès de Montpellier, j'avais tenu à préciser plus encore :

L'œuvre est... la somme de ses réalisations, elle est donc «partie» de ces interprétations (multipliées) de même que celles-ci sont « parties » de l'œuvre4.

Js - De même que la musicologie se découvre plurielle, non sans malaise, devant la multiplicité de ses méthodes et de ses objets d'étude, de même le musicologue frôle l'abîme lorsqu'il s'agit de définir " la musique » : L'Encyclopédie de Nattiez insiste clairement sur cet aspect dialectique, pluralité des musiques/unité de la musique. Selon vous, qu'estce qui constitue l'objet musical ?

JMB - À nouveau posée par la publication d'une Encyclopédie pour le XXI ${ }^{e}$ siècle (Actes Sud) cette infinité des accès me paraît la définition même de "l'objet " analytique musical, plus exactement, cette béance vertigineuse qu'est l'incessant déplacement de «l'interprété » du musical. Ce serait la somme des relations effectives et potentielles avec le sonore volontairement pensé-entendu comme différent des autres sons du quotidien d'un groupe humain et ayant une fonction spécifique, associée à certains actes de la vie de ce groupe. Je le présente de cette manière car nous savons qu'il existe un débat sur ce que signifie le mot « musique » pour chaque culture. Il s'agirait donc de l'ensemble des relations de sens établies de bien des manières différentes vis-à-vis d'une forme symbolique sonore, la somme de ces actes relatifs constituant le phénomène global pour l'espèce, à condition de rapprocher des situations qui ont des parentés fonctionnelles, toute relation étant évidemment analytique d'une manière ou d'une autre.

L'objet musical est l'objet $d u$ musical, au sens d'un «son » projeté dans un espace de mémoires, de temps interférés. C'est un territoire investi par un ou des écoutants, une 
zone fréquentielle dont la fréquentation induit une inter-pénétration qui diffuse ce que l'on pourrait observer comme "objet " dans la mesure ou toute "écoute", tout «entendement» qui le vise le transforme immédiatement. Ainsi, de cette relation, singulière chaque fois, demeure la relation elle-même qui implique deux moments mobiles et toutes leurs dérivées. C'est celle, en situation d'écoute, d'un multi-mémoriel réseau neuronal d'une complexité astronomiquement comparable à celle de l'univers ${ }^{5}$. L'objet serait une représentation en constant déplacement.

Ce moment que l'on nomme œuvre est un effet de sens, multisémique, souvent particulièrement dense à des niveaux très divers. Des auditeurs plus ou moins attentifs s'y adaptent et l'adaptent sans cesse, leur nombre dépendant de sa diffusion, des imprégnations dont elle a été susceptible à la faveur de médiations plus ou moins efficaces. Si elle est fixée sur un support («tableau-musique » rendu visible) sous forme de graphe couvrant une feuille en deux dimensions, la dérive est fonction des interprétations du sens de ces signes. Si le support a pérennisé l'une des interprétations sonores, de ses «restitutions" (qui inclut souvent une aspiration à la "vérité " originelle), de ses "exécutions", de ces "performances", c'est l'interprétation de l'auditeur qui la fera dériver.

Que ceci soit accepté ou non, l'analyse est infiniment plurielle du fait même du nombre de cerveaux qui se livrent à son " écoute ", chercheurs, mélomanes, tous " interprètes " d'une manière ou d'une autre, c'est-à-dire mettant en jeu un ensemble de processus de segmentation, repérage, regroupements, classements, comparaisons, évaluation par rapport à un savoir antérieur, mise en lien avec du sens dans tous les autres domaines associés de près ou de loin. Elle l'est à travers la diversité des paradigmes utilisés en musicologie " générale » - sans plus faire de distinction tranchée entre musicologie(s) historique(s), musicologie(s) analytique(s), ethnomusicologie, etc...

Js - Verriez-vous une alternative à l'opposition qui renvoie la constitution de la discipline musicologique soit à son champ d'étude (est musicologique toute étude prenant pour objet la musique), soit à sa méthode (seule la pratique de l'analyse relève de la musicologie)?

JMB - Ce sera précisément la thématique dévolue à la Société Française d'Analyse Musicale lors de notre prochain (et déjà) septième Congrès Européen, qui aura lieu en 2011 à Rome: "Corpus et méthodes ». Au-delà de la délimitation plus ou moins « accordée » de territoires spatio-temporels réunissant des ensembles de «moments » de pensée musicale, (d'ailleurs sujets, bien évidemment, à remaniements incessants : qu'est-ce que la «musique découpée en siècles » par exemple ?) il s'agit selon moi de savoir comment se dégagent et se déclinent de nouvelles appréhensions anthropomusicologiques à partir de l'ensemble des méthodes appliquées à des champs particuliers ou bien à plusieurs, voire, partiellement au moins, à tous.

\section{Que reste-t-il de l'un, du beau, du vrai ?}

Js - Face à une telle situation d'éclatement (de l'objet de recherche comme des méthodes de la discipline), tout invite à penser que la musique a défınitivement quitté le régime de l' « un » : la musique n'est pas une, et l'œuvre musicale elle-même connaît une déclinaison de ses modes d'être. Vous dites vous-même dans votre ouvrage sur Les écrivains et la musique au XVIIIe siècle ${ }^{6}$ que « le fossile n'existe pas en Musique. Aussitôt exhumé, il ne revit pas, il vit. [...] Même si l'on ne joue pas exactement la musique dont on a retrouvé les traces, les preuves, les vestiges ou les survivances locales, comme leur inventeur (singulier ou pluriel) les jouait, elle devient notre musique et fait partie de notre culture, plus encore sans 
doute qu'une amphore dans un musée. » Comment éviter alors le relativisme intégral ? La question de la valeur de « l'œuvre » reste-elle toujours pertinente?

JMB - L'évaluation s'applique à l'invention humaine. Je veux dire que toute pensée est celle - potentiellement - de chaque individu de cette espèce. L'Art de la Fugue, le Gagaku (japonais) tout comme les théories les plus récentes de l'astrophysique sont «parties » de la pensée de chacun, quand bien même chacun n'y a pas nécessairement accès. La vie est trop courte pour s'attacher à des effets de " divertissement » fugaces, modélisés, diffusés par des industries en inflation d'elles-mêmes, souvent à des fins, non avouées, de distraction qui interdisent la modification, la métamorphose, la mutation à la faveur de l'interprétation continue qui semble être l'essence de la vie : l'évolution.

Js - La musique oscillerait donc entre l'ordre de l'inventio et celui du souffle de l'Esprit?

JMB - Que la musique naisse d'une inspiration... on peut bien encore y croire - je lui préfère la notion de «court-circuit». Mais cette persistance de l'image d'un surgissement n'est nullement contradictoire avec l'ordre complexe d'une morphogénèse sonore. Le Quadrivium (regroupant musique, astronomie, géométrie et arithmétique), souvent cité dès lors que l'on évoque les liens intimes entre création musicale et conception des dimensions universelles que nous habitons à grand peine, nous rappelle simplement qu'à une époque pourtant très imprégnée de souffle divin, il n'était pas inquiétant de concevoir la pensée musicale et la pensée scientifique comme gémellaires. Il serait possible de le dire autrement: les musiciens sont des chercheurs d'inoui.

Js - L'analyse ne commet-elle pourtant pas une «réduction» de cet inouï en nous proposant un modèle de compréhension?

JMB - On s'inquiète de ce que la musique puisse être «modélisable »!... Mais comment conçoit-on des formes musicales, y compris les plus «romantiques» ou les plus apparemment improvisées d'entre elles, si ce n'est à l'aide de structures plus ou moins fines, qui sont mises en œuvres à divers niveaux de combinatoire plus ou moins subtiles? On n'amoindrit pas le plaisir de l'écoute lorsqu'elle est plus active encore, plus consciente de certains aspects du « poiétique », bien au contraire. Dit-on d'ailleurs que le «déchiffrement " des tableaux par des conférenciers, y compris lorsqu'ils se livrent à des analyses très fouillées des structures sous-jacentes, des procédures, des motifs d'élaboration, qu'ils privent leurs auditeurs-spectateurs d'une émotion intense face aux toiles? On sait mieux maintenant qu'émotions et invention sont des aspects liés de la pensée.

Il s'agit de chercher des "modèles réduits » selon l'expression utilisée par Gérard Assayag (Ircam). Ce sont des systèmes mettant en ordre des «objets trouvés » (acoustiques, rythmiques, timbriques, modaux, systémiques etc...) les regroupant, les classant et les reclassant sans cesse selon des paradigmes réinventés dont on espère qu'ils auront force heuristique.

En savons-nous davantage à propos des squelettes que sont les notations musicales, les différents modèles de partitions qui tentent de fixer une forme sonore comme la charpente de calcaire fixe la forme vivante, qu'à propos de celui d'une très ancienne humanoïde supposée, récemment découverte dans des gisements de tourbe en Allemagne? Et lorsque la forme vive nous est transmise par les relais des mémoires reproduisant l'identique d'une information complexe, ou bien lorsque seul l'enregistrement nous est livré, comment avons-nous accès à sa compréhension? En 
cherchant à constituer un squelette, en écrivant des manières de partitions possibles et en écoutant les témoignages divers de ceux qui ont connaissance du contexte dans tous les sens de ce terme, puis en superposant les unes et les autres afin de déceler des coïncidences. Et puis, il y a l'écoute elle-même, ce lacis interprétatif qui réinvente la forme sonorisée. La connaissance plus fouillée des processus en jeu dans notre cerveau permet d'y pénétrer plus avant, ainsi que de nombreux chercheurs s'y emploient. Autant d'« œuvres » que d'écoutes, simultanées, successives!

\section{Plaisir et complexité : de l'enseignement à la création.}

Js - L'Église catholique romaine a longtemps lutté contre l'envahissement du plaisir dans la musique. II fallait endiguer les passions dangereuses. Vous rappelez d'ailleurs à ce propos, devant la frilosité quant à l'usage du chromatisme? ${ }^{7}$ que l'Église interdisait l'usage de l'enharmonie héritée de la musique grecque antique, jugée trop sensuelle.

JMB - Il me semble, au contraire, que complexité et jouissance sont intimement liées. L'Eglise de la contre-Réforme pour ne citer que ce cas, "autorise " un débridement invraisemblable de toutes les stimulations de jouissances et même d'illusions de jouissance (trompe-l'œil de ciel sur plafonds plats, myriades de putti quasiment "gustatifs " lancés à l'assaut des vides, convocation dans des espaces limités de fragments architecturaux, sculpturaux, de matières, de simulations, d'ors, torsions de corps, drapés aux plis infinis etc.).

L'enharmonique (deux en un) ce pourrait être l'ange (asexué, double, multiple, « myriadique » ? à ceci près qu'il a souvent figure masculine dans ses représentations picturales) et sa musique - figurée à défaut de pouvoir l'entendre. Le castrat en serait un avatar humain, qui provoquerait, à l'écoute de sa voix, les mêmes effets réceptifs de sublimité. Les instruments ont été tenus à l'écart par une partie des responsables doctrinaires liturgiques du champ des fréquences, des timbres autorisés, ou bien admis sous conditions, selon l'époque. Il existe par exemple une musique très lente, basée sur des sons longuement tenus ou bien sur des cellules indéfiniment reproduites. Cet ison (le son tenu infiniment) serait, au fond, la seule musique «religieuse " (moines tibétains, sons très graves du pédalier de l'orgue, moines orthodoxes, didjeridoo, etc...) qui procure la jubilation d'une fusion entre la finitude et l'éternité.

Js - Contre une vision linéaire de la pensée musicale, et sans doute aussi contre tout un discours dominant autour d'une génération sérialiste, vous affirmez qu'il «n'y a pas Progrès en Arts (notion chère au XVIII siècle), mais des mutations. II y a la Musique. Et le plaisir musical. Notion fondamentale que beaucoup oublient souvent. » (p. 109)

JMB - C'est un texte ancien que je n'ai pas relu depuis... Diderot évoque l'idée de "mourir de plaisir». On pense immédiatement, dans ce contexte des pâmoisons baroques, en voie de transformation en défaillements post-héloïsiens, à la transverbération thérésienne ( «je meurs de ne pas mourir »...) cette «inondation» dont parle Julia Kristeva qui en rappelle une autre, dans le cerveau du musicien écoutant la musique: phénomène récemment mis à nu par un «suivi » des circuits neuronaux ainsi éveillés, qui s'apparente à un feu d'artifice mais également à la corde coupée, dans le texte sadien, au juste moment qui sépare la jouissance ultime de la mort.

Un seul exemple de relations très contradictoires entre pouvoirs institués et pouvoirs de l'acte artistique peut venir à l'esprit. Celui qui concerne ce que Pascal Boyer ${ }^{8}$ appelle 
les «corporations de lettrés ", fondant peu à peu un corps de doctrine, jusqu'aux théocraties, qui veulent contrôler tant la jouissance, ferment libératoire, que l'interprétation (dérives des codes, des structures ne varietur liées à des rituels) et, finalement, l'autonomie critique d'une pensée et leurs réactions pour le moins ambiguës, avec l'un des « véhicules » musicaux. Outre les velléités d'interdiction de la musique ou sa réduction à une seule forme autorisée, la manière dont l'orgue a été plus ou moins admis est emblématique. Mais n'est-il pas ce grand Pan polycalame venu d'une autre culture, dont l'accès aux plaisirs était souvent radicalement opposée, planté au cœur même de l'enclos consacré, puissance sonore formidable, instrument de jaculatio extraordinaire, donnant accès à une complexité timbrique, harmonique, contrapuntique, texturale, rythmique, ouvrant sur l'improvisation, l'invention dans l'instant et son potentiel de mutation de l'appréhension du musical (car c'est bien d'une succession de mutations déterminantes dont il s'agit de s'approprier les logiques, au cours d'une histoire sans cesse revisitée, en un processus personnel, singulier, de profond plaisir). Or, cette situation conflictuelle a suscité de violents rejets de la part de nombre de « clercs », qui ne sont pas encore relégués, loin s'en faut. Les plus habiles ont mis ce potentiel à leur service.

Cependant, nous sommes une espèce vouée à la complexité (à la « simplexité », comme l'écrit Alain Berthoz $z^{9}$ à la fois dans son histoire et pour réussir à survivre. L'activité réflexive connait ces niveaux d'interférences multipliées qui ramifient en tous sens, au cœur de la jouissance (une forme de jubilatio) la plus «intégrée ». Je ne fais pas de distinction entre pensée dite artistique et pensée scientifique : elle est "à l'œuvre » dans tous les cas et il est à espérer que le plus grand nombre d'individus puisse avoir accès à cet excès, à cette « expérience des limites ». N'est-ce pas l'une des conditions de la démocratie humaniste?

Lorsque Diderot, dans ses Leçons de Clavecin et principes d'harmonie par M. Bemetzrieder, évoque ainsi la formation musicale globale, il parle immédiatement du bonheur de jouer et de comprendre en même temps:

L'élève : «À quand ma première leçon?»

Le maître : « Il ne faut pas différer l'œuvre de son bonheur ; à l'instant »

Notre époque n'est certes plus celle-là. Pourtant, la dissociation instituée entre «théorie » et " pratique » n'a pas davantage lieu d'être qu'au XVIII siècle. S'ensuit, dans le livre du même auteur, la description d'un cours qui associe sans cesse pratique au clavier dans ce cas - et approche des codes et de la complexité de la structure. Puis, ce nouveau dialogue :

« Est-ce que vous ne recommandez pas d'exercer beaucoup »?

« Point du tout».

Diderot met en scène une enfant qui « touche du clavecin, six heures par jour, depuis six ans et qui ne sait rien ». Il stigmatise un apprentissage qui tournerait comme à vide. Et le maitre d'initier immédiatement sa jeune élève de clavecin aux "arcanes" de l'harmonie, de l'écriture, à la compréhension de la mise en forme, de la composition. J'ajouterai: la conscience de la globalité de la musique humaine, le plaisir de l'improvisation, du fait de jouer ensemble, la découverte de sa propre vocalité, le déchiffrage, l'appréhension des pouvoirs du sonore, de sa place dans les territoires sociaux et tant d'autres aspects encore. La question de l'efficacité est d'autant plus flagrante que les élèves des conservatoires contemporains (hormis, peut-être, une partie des enfants de musiciens) disposent de bien moins de temps qu'un enfant du 
XVIII ${ }^{e}$ siècle qui appartenait à des milieux lui rendant possible une formation artistique complète, suffisamment poursuivie dans le temps et souvent quotidienne. D'ailleurs la conception actuelle des temps scolaires est une aberration, ce que ne cessent de relever les psycho-pédagogues, les chronobiologistes, entre autres. De plus, la connaissance du répertoire global s'est considérablement étendue à l'ensemble de la musique du Sapiens. Il faut donc choisir selon des impératifs pédagogiques, techniques, mais aussi en fonction d'un temps très limité, ce qui signifie que les professeurs sont tenus de faire connaître aux élèves ce qui leur semble le plus important parmi ces répertoires, une part des œuvres de tous temps et lieux s'imposant en tout état de cause, quels que soient la formation, les orientations, les présupposés personnels.

\section{Js - Comment analysez-vous la situation de la création contemporaine?}

JMB - Diffère-t-elle tant de celle qui prévalait aux XVII et XVIII ${ }^{e}$ siècles, moment de curiosité prodigieuse, qui voit fleurir des syncrétismes esthétiques: influences multiples dans toute l'Europe entre musique de danse, musique de concert, de scène et vouée au culte, musique des Jésuites dans l'Empire chinois, qui emprunte à la " modalité », aux instruments " endémiques » jusque dans le rituel catholique, musique en Amérique du Sud, utilisant des instruments indiens et africains? La création sous tous ses aspects (artistique et scientifique) était très fortement financée par les pouvoirs des Cours. Une émulation entre eux produisait un effet favorable, propice au développement d'une pensée de la transmission à l'intention de nouveaux venus à l'art musical. Des publics se constituaient (opéras, Concert spirituel, improvisations à l'orgue etc.) qui appréciaient à ce point la nouveauté qu'ils n'aimaient pas ré-entendre! Voltaire avait cependant bien envisagé le décalage toujours patent entre l'invention de l'inouï et des «mélomanes» plus inquiets, en précisant que c'était affaire d'une cinquantaine d'années pour qu'ils s'y accoutument. Seul peut-être Rousseau eut été réfractaire à la nouvelle musique orchestrale. A un siècle de distance, Debussy est-il désormais «assimilé » aux mémoires diverses? Et quel Debussy? Quelle œuvre? Par qui ? De quelle manière?

Quant à la musique des cinquante dernières années... La plupart des adolescents, privés d'une formation artistique qui serait considérée véritablement comme un «fondamental " sont embusqués derrière leurs écouteurs, ayant "opté » pour un «objet» présenté comme signe d'appartenance identitaire d'une classe d'âge. Ils ignorent la musique réellement " actuelle», sauf lorsque d'aventure, ils peuvent y avoir accès à la faveur d'une "expérience » d'écoute à l'école, lors d'un déplacement au concert ou lors de jeux d'improvisation.

On pourrait distinguer, de manière très large : la musique des «trouveurs " (c'est un mot qui me semble très adéquat et «transculturel») descendants des premiers chamanes qui accompagnent les groupes de Sapiens depuis leur "apparition ", c'est à dire celle de l'histoire musicale humaine très inventive, qui ne craint pas l'inouï, la musique dite de «tradition orale» et ses "prolongements" dans la musique dite "savante", la musique de grande diffusion commerciale, et un courant de musique « syncrétique » qui emprunte à tout ce qui est accessible en inventant, en tous lieux du monde, dans le cours d'une globalisation des savoirs. Concrètement, je dirais qu'outre le fait d'admettre enfin la pratique artistique au sein des "fondamentaux» à tous niveaux de l'enseignement dit "général», il faudrait encourager les festivals qui incluent systématiquement des « créations » au cœur de programmes thématiques bien pensés, favoriser la musique de création par son inscription sur des supports divers 
aisément accessibles, stimuler la collaboration entre compositeurs et réalisateurs (dépasser l'indigence des "bandes-sons » et des violons de synthèse!) développer la pratique des compositeurs/trices "en résidence» dans tous les établissements de Formation artistique spécialisée ou de formation générale, les cours d'histoire des arts, densifier le niveau d'appréhension de la complexité musicale globale afin de rendre chacun exigeant, critique, capable de jugement esthétique, ce qui suppose une volonté politique de généraliser la pratique artistique. Il conviendrait d'ajouter: dans les meilleures conditions possibles, sans céder aux facilités infructueuses et même contreproductives, aux solutions moins coûteuses.

Js - Dans votre ouvrage Les écrivains et la musique au XVIII siècle, vous retenez comme l'un des aspects majeurs de cette période la recherche d'une rationalisation, d'un universalisme dans le discours musical, qui se traduit non seulement par la recherche et la fixation d'un langage commun (la tonalité), mais aussi par la volonté d'uniformiser le temps (chronomètre) et le son (tempérament): "Partir à la découverte de l'aspect «physicomathématique», formel, de la production des sons, c'est tenter du même coup pour Diderot de lui conférer un pouvoir universel au travers des spéculations concernant l'aspect combinatoire - du reste fort complexe - de la Création musicale. » (p.28) Or, le XXe siècle semble être sous l'effet d'une tendance inverse, à la fois marqué par l'éclatement des langages, la complexification des dispositifs de création, la marginalisation des règles de l'art, de plus en plus individuelles - d'où l'accompagnement fréquent de l'œuvre par un discours théorique. Faut-il voir derrière cela le symptôme d'un refus de l'universel, la rupture avec une certaine idéologie de la musique, voire, comme le suggère Menger dans Le paradoxe du musicien, l'affirmation d'une spécialisation de l'art délibérément réservé à une élite?

JMB - Haute culture ? Musiques « actuelles » ?... Il y a une culture de l'être humain « actuel ». Il y a des cultures « endémiques » plus closes, plus repliées. Il y a une culture mondialisée contemporaine, dupliquée à l'intention de couches qui ont beaucoup moins accès à la culture de l'homme sous tous ses aspects. Mais à toute époque, le fait d'appartenir à l'une des castes ou l'un des groupes au pouvoir confère la possibilité de considérer la culture de l'autre plus aisément. Ainsi, par exemple, de l'attention portée par une partie des théologiens de la Curie aux textes grecs ou d'autres cultures, la constitution de cabinets de curiosité, de collections d'art par les cardinaux appartenant à des familles justement très "cultivées", aristocratiques et fortunées. Plus récemment : la conception d'un Musée du Louvre dans le golfe persique ne rend-t-il pas compte de ce désir, commun à toutes les époques, de maîtriser l'ensemble de la culture humaine ? Il existe également ce souci musicologique de constituer le répertoire global des répertoires de musique de tradition orale ou partiellement orale (le travail de Bela Bartok ayant été, en ce sens, un fer de lance) le classement de pans entiers de cette mémoire musicale en Patrimoine immatériel de l'humanité induisant un processus évaluatif collectif tout à fait remarquable.

Ainsi voyons-nous plus clairement un enjeu qui serait de réserver la culture humaine à ceux qui peuvent y avoir accès dans ses parties «mémorielles » comme créatrices, et laisser le « marché » gérer une culture massifiée, dupliquée, réduite, minimale. Il existe la culture artistique/esthétique/philosophique/scientifique (peut-on séparer?) et tous les processus d'appropriation sociale. Prétendre que la première est totalement indépendante serait aussi absurde que de prolonger les catégories du type 'musique d'une religion donnée' dans les histoires de la musique. Dans l'Encyclopédie pour le XXI ${ }^{e}$ siècle, par exemple, aura-t-on remarqué que dans le tome consacré aux musiques du monde, le classement se fait selon des critères spatiaux, hormis pour une catégorie 
musicale nommée 'Musique de l'Islam' : signe du temps, intervention circonstanciée du politique dans le champs musicologique? Des compositeurs marocains, tunisiens par exemple, - pas nécessairement estampillés comme «musulmans», ni chrétiens, ni même nécessairement croyants, d'ailleurs - écrivent la musique de leur temps, qui appartiennent au groupe de ceux que l'on nomme 'contemporains' en ajoutant souvent 'savants'.

Pour résumer cette question touffue, je pense qu'un individu singulier appartenant à l'espèce actuelle du Sapiens, est potentiellement ou effectivement confronté à l'ensemble multiple des inventions musicales uniques, chacune comportant à la fois une manière d'inouï ainsi qu'une part de complexité, en elle-même, et surtout si on la rapporte à l'ensemble de la complexité de la musique humaine depuis ses origines, en ses innombrables mutations.

Js - Dans Le paradoxe du musicien, Menger met en évidence la situation paradoxale de la création contemporaine, une avant-garde revendiquant la complexité, nécessairement soutenue par le pouvoir public et à ce titre devenue « art officiel »... Faut-il redouter un effet neutralisateur dû à la prise en charge de la vie artistique par les politiques publiques?

JMB - Son tout dernier ouvrage (Le travail créateur, Gallimard-Seuil-HE 2009) qui comporte un sous-titre très éloquent : s'accomplir dans l'incertain, aborde la question de manière quelque peu différente. Art «officiel»? Le paradoxe n'est-il pas que des politiques peu au fait de culture artistique, soutiennent un art qui peut être considéré comme étant réservé aux couches les plus aisées (alors même qu'une partie de celles-ci devient de plus en plus inculte) et qu'ils peuvent afficher un goût pour l'art dit « populaire » - en tous cas, de fait, « réservé » à ceux qui n'ont pas accès, pour bien des raisons, à la « pensée à l'œuvre » et qui seraient pourtant très passionnés si on leur en proposait la fréquentation, ainsi que le montrent de manière incontestable, pour ne citer que des cas hexagonaux, l'expérience remarquable de la Folle Journée de Nantes, le Prix des lycéens, celle menée depuis des années à l'Opéra Bastille avec des groupes de jeunes adolescents, ou bien par telle ou telle structure de création «électroacoustique ». C'est uniquement une affaire d'initiation et de moyens mis en jeu pour la rendre largement possible. Le plus étonnant n'est pas que les pouvoirs publics "subventionnent » l'étrave de l'invention mais qu'ils aident également la musique de grande diffusion commerciale - qui se soutient fort bien elle-même à travers les vecteurs internationaux habituels - par exemple: une grande part des musiques " amplifiées » dont on sait le ravage (irrémédiable, notamment sur l'oreille interne) qu'elles peuvent entraîner et l'indigence qu'elles véhiculent (boîtes à rythmes, échantillons pauvres, boucles etc.).

On ne saurait déplorer que des monarques « éclairés » aient soutenu la créativité de la pensée artistique, philosophique, scientifique-technologique alors que nous héritons d'un colossal patrimoine de "commandes». On pourrait également citer le cas de l'empereur Frédéric II Hohenstaufen, étonnant mécène du XIII ${ }^{\mathrm{e}}$ siècle, polyglotte érudit, curieux d'art et de science, qui avait réussi à mener une croisade pacifique à une époque de fureurs guerrières incessantes. Le mécénat d'entreprise est-il préférable parce que ses «choix » seraient plus libres? Les effets de mode peuvent s'imposer de toute part. Je dirais plutôt qu'il n'y a pas, de ce point de vue, d'économie privée et publique. On le voit bien lorsque des pouvoirs d'état reprennent la maîtrise financière, ne fut-ce que pour un temps limité ; on l'a constaté récemment lorsque les puissances publiques doivent compenser les gouffres de «pertes » causés par des structures 
qualifiées de "privées». Il est également possible de penser à des conjonctions de financements dans le cadre de processus de formation, pour revenir à un thème que j'ai largement abordé : lorsque les pouvoirs publics à divers niveaux, ainsi que des manières de mécénats contribuent à un projet de formation artistique pour développer ensemble festivals, concerts comportant des entrées gratuites pour des classes, achat d'instruments pour les orchestres à l'école, ou encore interventions d'artistes dans les cursus d'enseignement. D'une manière plus générale, je pense il s'agit de permettre le dégagement d'une lucidité collective, d'une vivacité d'invention face aux problèmes suscités par notre espèce.

\section{La lutte : pour un régime du pluriel}

Js - La place de la musique dans la Cité est problématique depuis l'antiquité, on se souvient notamment du projet platonicien de la République. Penser la musique ne se résume donc pas à penser les seules règles de l'art mais bien aussi la fonction du musicien dans la société et son rapport à la collectivité. D'un point de vue contemporain, quel sens attribuez-vous à cette relation musique/société dans l'activité du musicien ? Peut-on parler d'engagement?

JMB - C'est un véritable engagement dans la mesure où la formation artistique dans l'enseignement dit "général », c'est-à-dire pour chaque enfant, est encore largement déniée, malgré les déclarations et les tentatives multiples. Elle est déniée en ce qu'elle représente de plus vital. Elle est submergée par un flot de musique "réifiée », par les eaux tièdes lénifiantes ou bien acides d'un flot continu dans l'espace public aussi bien que privé (à peine privé d'ailleurs). Et rendre l'autre «sourd » - à 110 décibels et en MP3 c'est-à-dire en compensant la perte de la subtilité du son par l'intensité en quelque sorte - est manifestement encore une clé du pouvoir. Alors, le rendre à son désir de musicien, lui permettre d'avoir une pensée singulière, c'est en soi un début de résistance.

Js - Vous revendiquez l'ouverture, le décloisonnement de «l'espace musical» (cf. notamment Préface à Diderot). L'une de vos tentatives dans cette optique se concentre autour de la confrontation ou mise en rapport de l'image et du son. En introduisant ainsi l'image dans le processus du concert, vous modifiez la teneur de ce dispositif. Est-ce une réaction contre l'héritage du concert bourgeois figé par le XIXe siècle ? Pourquoi ce privilège à l'image?

JMB - Nous savons mieux à quel point perception du sonore et du visuel peuvent être liés dans le lacis neuronal. D'ailleurs, le concert ne suscite-t-il pas de nombreuses visions d'un autre ordre, mémoriel, en particulier dans toutes les couches plus ou moins inconscientes ? Les liens étroits entre visuel et sonore sont très anciens, l'opéra étant l'un des jalons de cette association qui connaît de nombreuses tentatives jusqu'aux « installations ». J'avais imaginé un système informatisé destiné à être placé au cœur d'un labyrinthe et qui aurait placé les auditeurs face à un mobile créant sans cesse de nouvelles «polytonies ». J'ai proposé, tout récemment, avec Jean-Marc Chouvel, compositeur, ingénieur, peintre-plasticien, une collection de livres ayant pour objet la mise en perspective des actes artistiques correspondant à une polyvalence chez un même individu (laquelle n'étonnait pas les Baroques mais heurte les codes actuels d'une hyper-spécialisation, cependant qu'elle s'avère pourtant scientifiquement indispensable). Or, plus nombreux qu'on le pense, pour ne citer que cette " catégorie ", sont les compositeurs qui peignent, dessinent, sculptent, photographient ou bien 
utilisent les moyens informatiques pour inventer parallèlement ou même conjointement, du «visuel». Daniel Humair et Brian Ferneyhough en font partie. Beaucoup plus généralement, cette collection interrogera l'ensemble des relations entre l'acte de voir et celui d'entendre sous tous ses aspects. On connait les remarquables travaux du compositeur Jean-Yves Bosseur à ce sujet. Des professeurs de départements de musicologie s'y consacrent également, dont Michèle Barbe, à la Sorbonne.

Js - Votre importante activité éditoriale est-elle une autre façon pour vous d'expérimenter l'ouverture de l'espace musical?

JMB - La réponse serait trop longue, mais je confirme au moins ceci : depuis mes premières recherches pédagogiques, enfoui dans les partitions de la BNF, et celles sur le XVIII ${ }^{\mathrm{e}}$ siècle, il est certain que l'espace musical était très largement ouvert pour moi et qu'il l'est plus encore maintenant. Je citerai ici l'expérience éditoriale que j'ai tentée, assez jeune, en réalisant une collection commune à Six Editeurs musicaux, Carrousel, vouée à des œuvres de compositeurs (l'utopie a parfois des ailes icariennes, qui se sont brûlées) et celle, plus récente, d'un site Internet consacré à la création musicale, et qui ouvrirait la curiosité à la faveur d'un accès plus immédiat aux partitions : ce sont là deux expériences de « dégagement » d'espaces encore trop réservés.

Js - Au Conservatoire Hector Berlioz, vous enseignez l'écriture-composition : il n'y a pas lieu, pour vous, de compartimenter les disciplines techniques que sont l'analyse, la composition, l'écriture, l'harmonie?

JMB - On n'apprend sans doute pas à « composer » stricto sensu, c'est un désir qu'on a d'abord. On apprend des contextes, des conditions nécessaires au développement d'une pensée musicale. On apprend la découverte de sa propre singularité. C'est cette singularité qu'il est précieux de mettre en jeu. Autrement dit, un cours d'écoutetranscription-mémorisation-écriture-reproduction de modèles-improvisationinvention-composition suppose le plus possible d'accès à des cohérences diverses telles qu'elles ont été conçues par des humains depuis dix mille ans au moins. Parmi ces cohérences stylistiques, il faut aller aux plus mémorables, aux plus denses, aux plus subtiles, complexes à tous égards, car la vie est courte et parce que ce qui survit après le temps, ce sont les traces de l'art le plus ambitieux (des pyramides !...). On apprend les conditions d'une curiosité pour l'acte artistique en tous moments de l'histoire globale, les conditions d'une considération attentive aux contextes uniques fondateurs de cohérences, des pensées successives qui ont pu donner lieu au plus inventif, aux contextes de pensée philosophique, scientifique, aux contextes symbolico-mythiques souvent associés, aux mutations de cette pensée en acte permanent, donc une nécessaire polyvalence de l'écoute, à la recherche des plaisirs inouïs. Cette ambition existait particulièrement à travers la porosité extrême de la période dite "baroque ", foisonnante, lorsque toute l'Europe n'était qu'emprunts d'un lieu à l'autre.

On apprend les conditions d'un désir de poursuivre une histoire très longue : il s'agit de pouvoir la connaître, mais également lorsque le chemin, patient, détourné, mutant, n'a pas été parcouru, depuis les petits moines - «enveloppes soufflées » incarnant le chant orné, "cantillé », de l'inspiration - jusqu'à Charles Ives, depuis Ockeghem jusqu'à Kagel, de Gesualdo à ceux que l'on nomme «futuristes ", de faire écouter la création la plus récente, de faire réfléchir aux modes incantatoires, compensatoires, circulaires, à ces répétitions (du grand Crocodile des mythes Dogons, frappant de sa queue sur la Terre) qui ont pris la forme terriblement morbide du marteau-piqueur de la techno, de 
l'intensité maximale qui a pour conséquence des surdités définitives. Autrement dit, l'on se hasarde sur un territoire musical hanté par les psittacismes, les impasses, l'univocité, les refuges identitaires. Une fausse-monnaie...(c'est bien le moins en ces temps de "virtualité » mondialisée !...). Il conviendrait de préciser qu'il s'agit d'un mode substitutif : c'est, à défaut, de fournir à chacun les moyens les plus larges, les plus complets, les plus denses possibles d'être un musicien devenant professionnel ou un musicien qui poursuit son chemin parallèlement à une ou plusieurs autres activités socialisées.

Un cours d'écriture-composition-analyse-culture suppose tout cela. Serait-ce trop ambitieux? Il faut ensuite prévoir toutes les situations particulières, mais à partir de cette nécessité, toujours.

\section{NOTES}

1. Colloque tenu les 7 et 8 novembre 2008 à la Cité de la Musique/CNSMDP.

2. Colloque tenu les 6, 7 et 8 novembre 2008 à l'Université de la Sorbonne

3. Cf. BARDEZ, Jean-Michel, Les écrivains et la musique au XVIII ${ }^{e}$ siècle, t. 3, Philosophes, encyclopédistes, musiciens, théoriciens, Paris, Slatkine, 1980.

4. Analyse et création musicales, Paris, L'Harmattan, 2001.

5. Cf. CHANGEUX, Jean-Pierre, Raison et Plaisir, Paris, Odile Jacob, 2002.

6. Les écrivains et la musique au XVIII ${ }^{e}$ siècle, t. III, Philosophes, encyclopédistes, musiciens, théoriciens, Paris, Slatkine, 1980, p. 114.

7. Diderot et la musique, p. 59 : « est-ce qu'il se souvient de l'ancienne interdiction de l'Eglise ? Estce parce que la musique deviendrait trop sensuelle, trop agréable? Parce qu'on y perdrait ainsi le sens raisonnable de l'imitation de la nature? »

8. BOYER, Pascal, Et l'homme créa les dieux, Paris, Gallimard, 2003.

9. BERTHOZ, Alain, La simplexité, Paris, Odile Jacob, 2009.

\section{RÉSUMÉS}

Dans cet entretien, Jean-Michel Bardez, président de la Société Française d'Analyse Musicale et professeur d'écriture-analyse-composition au Conservatoire H. Berlioz (Paris X), développe un point de vue original sur l'état disciplinaire de la musicologie contemporaine. Il propose une réflexion sur le statut et la place de l'analyse dans le double champ de la recherche et de la pédagogie en conservatoire. Cette réflexion est l'occasion de revenir sur l'évolution de sa conception d'un "espace musical ouvert» - un véritable plaidoyer pour le dialogue des 
disciplines et l'affirmation d'une responsabilité croisée des enseignements artistiques et scientifiques sur le plan institutionnel.

As President of the French Musical Analysis Society and analysis-composition teacher in the Conservatoire H. Berlioz (Paris X), Jean Michel Bardez develops an original point of view on the state of contemporary musicology. He ponders on the status of analysis in both fields of musicological research and pedagogical activity in Music Conservatories. This is an occasion for him to come back to his conception of an "open musical field" - a pledge for more dialogue between disciplines and for the assertion of a shared responsability of scientific and artistic institutions.

\section{AUTEUR}

\section{JULIEN SÉGOL}

Ancien élève de Sciences-Po Paris et de l'Ecole des Hautes Etudes en Sciences Sociales, Julien Ségol est également titulaire d'un 1er Prix d'Esthétique du Conservatoire National Supérieur de Musique et de Danse de Paris. 